reagent. Their findings further suggest that for the paucibacillary type of samples, such as CSF, less sample reagent might be necessary.

These findings need to be confirmed independently and should be combined with testing of the tuberculocidal effect at lower sample-to-sample-reagent ratios before they can be recommended for clinical use. We hope that other groups will follow the example of R.F. Luo and co-workers and evaluate different protocols for different sample types, so that Xpert testing on nonrespiratory samples can be optimised.

0 @ERSpublications

Improved, evidence-based standard operating procedures for processing CSF samples needed to optimise TB detection http://ow.ly/ytgfc

Claudia M. Denkinger ${ }^{1,2,3}$ and Madhukar Pai ${ }^{1,2}$

${ }^{1}$ McGill International TB Centre, McGill University Health Centre, Montreal, Canada. ${ }^{2}$ Dept of Epidemiology, Biostatistics, and Occupational Health, McGill University, Montreal, Canada. ${ }^{3}$ Division of Infectious Disease, Beth Israel Deaconess Medical Centre, Boston, MA, USA.

Correspondence: Claudia M. Denkinger, Dept of Epidemiology, Biostatistics, and Occupational Health, McGill University, 1020 Pine Ave West, Montreal, QC H3A 1A2, Canada. Email: claudia.denkinger@mail.mcgill.ca

Received: June 112014 | Accepted after revision: June 122014

Conflict of interest: Disclosures can be found alongside the online version of this article at erj.ersjournals.com

Acknowledgements: We would like to acknowledge that we have collaborated with R.F. Luo and N. Banaei (both Clinical Microbiology Laboratory, Stanford University School of Medicine, Palo Alto, CA, USA) on other projects.

\title{
References
}

Denkinger CM, Schumacher SG, Boehme CC, et al. Xpert MTB/RIF assay for the diagnosis of extrapulmonary tuberculosis: a systematic review and meta-analysis. Eur Respir J 2014; 44: 435-446.

2 World Health Organization. Automated real-time nucleic acid amplification technology for rapid and simultaneous detection of tuberculosis and rifampicin resistance: Xpert MTB/RIF system for the diagnosis of pulmonary and extrapulmonary TB in adults and children. Geneva, WHO, 2013.

3 Weyer K, Mirzayev F, Migliori GB, et al. Rapid molecular TB diagnosis: evidence, policy making and global implementation of Xpert MTB/RIF. Eur Respir J 2013; 42: 252-271.

4 World Health Organization. The use of the Xpert MTB/RIF assay for the detection of pulmonary and extrapulmonary tuberculosis and rifampicin resistance in adults and children. Geneva, WHO, 2014.

5 World Health Organization. Xpert MTB/RIF implementation manual. Technical and operational "how-to"; practical considerations. Geneva, WHO, 2014.

\section{Importance of concomitant local and systemic eosinophilia in uncontrolled asthma}

To the Editor:

We read with interest the comprehensive, combined retrospective and prospective study on eosinophilia in asthma by SCHLEICH et al. [1]. As compellingly demonstrated by SCHLEICH et al. [1] concomitant elevation of sputum and blood eosinophil numbers is an important factor in poorly controlled asthma. These data agree with the view that both local airway "inflammation" and blood eosinophilia contribute as risk factors in asthma [2]. However, there are additional eosinophil features, other than just counts, that characterise asthma. Here we draw attention to the potential roles of primary lysis/necrosis of eosinophils as blood biomarkers and bronchial-pathogenic mechanisms, especially in uncontrolled asthma.

SCHLEICH et al. [1] briefly discuss previous findings on eosinophil numbers reported by VOLBEDA et al. [3]. However, a principal message of the latter study concerned association between poor asthma control and "activated eosinophils" and loss of "epithelial intactness", respectively, in bronchial biopsies. It emerged that the eosinophils had been activated by primary lysis that results in the spilling of toxic protein-releasing free eosinophil granules (FEGs) in the bronchial tissue $[3,4]$. 
Furthermore, contrasting the significant associations with FEGs, poor asthma control was not associated with the number of eosinophils in sputum or bronchial tissue $[3,4]$.

The observations by VOLBEDA et al. [3] support pathogenic roles of primary eosinophil lysis-produced, release-competent FEGs. Intriguing is the potential of this mechanism to derange the epithelial lining and evoke inflammation and remodelling as a consequence of exaggerated epithelial repair [5]. Inflammation should probably be equalled to inflammatory activity rather than the number of eosinophils.

MUNIZ-JUNQUEIRA et al. [6] demonstrate that blood eosinophils undergo lysis (plus spilling of FEGs) more readily the more severe the asthma is. Unprecedentedly, the pro-lysis propensity distinctly separates three levels of severity amongst children with acute asthma exacerbations [6]. Hence, proclivity of blood eosinophils for primary lysis may biomark severe asthma.

Inferentially, eosinophils would be primed for cytolysis before arriving in severely diseased bronchi. Priming may be produced by interleukin (IL)-5, which switches pro-apoptosis responses into primary lysis, as reviewed in PERSSON and UlLER [7]. We think such switching is important as: 1) it may explain the enigmatic absence of apoptotic eosinophils in diseased airways; and 2) anti-IL5 drugs may owe part of their anti-asthma effects to the inhibition of pro-lysis priming.

Furthermore, bronchial wall FEGs and clinical asthma were not reduced in anti-IL5-treated asthmatics despite the inhibition of sputum and blood eosinophils [8]. Hence, additional priming factors are likely to be involved in eosinophil lysis in asthma.

Hargreave's research group reported that FEGs in sputum distinguish severe exacerbations of asthma from stable asthma, and demonstrated the inhibition of FEGs at clinical improvement by systemic corticosteroids [9].

However, there are limitations with the sputum approach. Potentially underestimating the frequency of eosinophilic asthma, occurrence of intact and lytic eosinophils/FEGs in sputum does not always reflect bronchial wall events. For example, obesity-asthma exhibits bronchial tissue FEGs; in these patients, who lack sputum eosinophilia, lysis would have prevented tissue eosinophils from migrating into the bronchial lumen.

Hence, lysis of tissue-dwelling eosinophils may partly explain the lack of sputum eosinophilia in patients who nevertheless suffer from eosinophilic asthma [7].

Primary eosinophil lysis involves chromatolysis, cell membrane rupture, release of FEGs (in turn releasing toxic proteins and cytokines) and nuclear and cytosolic danger molecules [7]. Asthma-relevant stimuli, including allergens and microbial factors, produce prompt eosinophil lysis. Abundance of FEGs in lethal asthma, severe aspirin-sensitive asthma, severe late-onset asthma and asthma exacerbations, reflect extensive eosinophil lysis in these patients and the lack of inhibition of eosinophil lysis by standard corticosteroid treatment (as reviewed in PERSSON and UlLER [7]).

Genomics may now be combined with other omics and systems-biology approaches in attempts to find molecular signatures of asthma [10]. However, progress in understanding asthma still relies on developments using classical research as evidenced by SCHLEICH et al. [1]. We propose that propensity for, and execution of, primary lysis of eosinophils in blood and bronchi, respectively, needs attention in studies of severe asthma.

○ @ERSpublications

A novel drug target and biomarker, regulated necrosis of eosinophils emerges as a central mechanism of severe asthma http://ow.ly/zdbV6

Carl Persson ${ }^{1}$ and Lena Uller ${ }^{2}$

${ }^{1}$ Dept Clinical Pharmacology, Laboratory Medicine, Lund University Hospital, Lund, Sweden. ${ }^{2}$ Dept Experimental Medical Science, Unit of Respiratory Immunopharmacology, Lund University, Lund, Sweden.

Correspondence: Carl Persson, Dept Clinical Pharmacology, Laboratory Medicine, Lund University Hospital, S-22185 Lund, Sweden. E-mail: Carl.persson@med.lu.se

Received: June 022014 | Accepted: June 022014

Conflict of interest: None declared.

\section{References}

1 Schleich FN, Chevremont A, Paulus V, et al. Importance of concomitant local and systemic eosinophilia in uncontrolled asthma. Eur Respir J 2014; 44: 97-108.

2 Malinovschi A, Fonseca JA, Jacinto T, et al. Exhaled nitric oxide levels and blood eosinophil counts independently associate with wheeze and asthma events in National Health and Nutrition Examination Survey subjects. J Allergy Clin Immunol 2013; 132: 821-827.e1-e5. 
3 Volbeda F, Broekema M, Lodewijk ME, et al. Clinical control associates with measures of airway inflammation. Thorax 2013; 68: 19-24.

4 Fattahi F, Volbeda F, Broekema M, et al. Authors' response to Persson C: primary lysis/necrosis of eosinophils and clinical control of asthma. Thorax 2013; 68: 295-296.

Persson C. Primary lysis of eosinophils in severe desquamative asthma. Clin Exp Allergy 2014; 44: $173-183$.

Muniz-Junqueira MI, Barbosa-Marques SM, Junqueira LF Jr. Morphological changes in eosinophils are reliable markers of the severity of an acute asthma exacerbation in children. Allergy 2013; 68: 911-920.

Persson C, Uller L. Theirs but to die and do: primary lysis of eosinophils and free eosinophil granules in asthma. Am J Respir Crit Care Med 2014; 189: 628-633.

8 Flood-Page PT, Menzies-Gow AN, Kay AB, et al. Eosinophil's role remains uncertain as anti-interleukin-5 only partially depletes numbers in asthmatic airway. Am J Respir Crit Care Med 2003; 167: 199-204.

Pizzichini MM, Pizzichini E, Clelland L, et al. Sputum in severe exacerbations of asthma: kinetics of inflammatory indices after prednisone treatment. Am J Respir Crit Care Med 1997; 155: 1501-1508.

10 Chung KF, Wenzel SE, Brozek JL, et al. International ERS/ATS guidelines on definition, evaluation and treatment of severe asthma. Eur Respir J 2014; 43: 343-373.

Eur Respir J 2014; 44: 1096-1098 | DOI: 10.1183/09031936.00100414 | Copyright (CERS 2014

\section{From the authors:}

We thank C. Persson and L. Uller for their interesting correspondence regarding our recent research paper on the link between systemic and airway eosinophilia and asthma control [1].

As there has been a huge controversy on the role of eosinophils in asthma, the purpose of our paper [1] was to report our clinical experience on the relationship between eosinophils and asthma. In our study the link between eosinophil counts and asthma control is significant but not so strong, which is in keeping with the concept of concordant and discordant disease when relating symptoms to inflammation [2]. In fact only airway eosinophilia was directly associated with poor Asthma Control Questionnaire, with an $\mathrm{r}$ coefficient of 0.16 , while the blood cell count did not. As those patients who combined high blood and sputum eosinophils had worse asthma control, our interpretation is that blood eosinophils contribute to mount an intense airway eosinophil infiltration. The role of airway eosinophils in poor asthma control is further supported by the fact that they contribute in determining the level of bronchial hyperresponsiveness, a hallmark of asthma pathophysiology $[3,4]$. Having said this, we entirely agree that just looking at cell counts does not provide a complete picture of the cell role in pathophysiology. As strongly suggested and convincingly argued by C. Persson and L. Uller, primary eosinophil lysis in the airway is likely to be an essential contributor to the intensity of airway eosinophilic inflammation and, thereby, poor asthma control. Therefore, the relationship with asthma control could have been stronger if we had looked at eosinophil activation. As stated in our paper we also recognise that some patients who were called noneosinophilic, based on eosinophils contained in the airway lumen, may have been misclassified due to the persistence of eosinophils in the airway wall and engaged in the scavenging process by macrophages [5].

We would like to emphasise again that behind the results reported here our strategy was to really improve asthma care and management in a university hospital. Very much influenced by the letter by PAVORD et al. [6] and the study by GreEN et al. [7], 10 years ago we set up an asthma clinic in which we embarked on inducing sputum as a routine practice to monitor eosinophilic inflammation and adjust inhaled corticosteroids (ICS) prescription accordingly. For centres experienced in using induced sputum the recent European Respiratory Society/American Thoracic Society guidelines recommends that induced sputum and clinical criteria be used to guide treatment in adults with severe asthma, rather than by clinical criteria alone [8]. Although detailed treatment adjustment based on sputum cell count was left at the discretion of the clinician, we found in our prospective cohort a reduction in exacerbation rate by $42 \%$ (from 0.86 per patient per year to 0.50 per patient per year) in the year following the visit to asthma clinic. Though we are aware this finding has to be interpreted with caution because of the recall bias, we feel that it is reassuring, even more so it was not subordinated to a huge increase in ICS prescription. In addition it was reassuring that the patients for whom the clinician did not feel the need to prescribe an ICS after their asthma clinic visit, did not report any exacerbation in the following year. This suggests that using the mere cell count to assess inflammation in clinical practice may still carry some value and help phenotype asthma patients [9]. This assumption was indeed further supported by the DREAM study results where the efficacy of mepolizumab was partly dependent on the blood eosinophil count [10].

@ERSpublications

Assessing airway and blood eosinophilic inflammation in clinical practice helps to phenotype asthmatics http://ow.ly/zdhyE 